\title{
INCREASING OF STIFFNESS OF DOUBLE-ACTING PNEUMATIC CYLINDER
}

\author{
A. J. Czmerk
}

BUTE, Department of Mechatronics, Optics, and Engineering Informatics, Bertalan Lajos str. 4-6., 1111, Budapest, Hungary e-mail: czmerk@mogi.bme.hu

\begin{abstract}
Position keeping properties of actuators depend highly on their stiffness. The low stiffness of pneumatic systems is well known, which is disadvantageous during positioning and when a position has to be kept. It is one of the reasons why hydraulic systems have advantages over pneumatic systems when it comes to positioning tasks. This paper deals with the analysis of the passive stiffness of a double-acting cylinder. The stiffness of pneumatic cylinders is derived as a function of chamber pressures determined by the bulk modulus of pneumatic systems. The stiffness of a passive system of the chambers is written by the pressure change through small displacement of the piston and as a result of the restoring force. In case of the passive system the effect of the valve actuation is neglected. From the deduction it is clear that the stiffness of a cylinder changes along the stroke, dead volumes at the end positions play a relatively important role. The results are compared with the restoring forces of the piston seals and other sealing elements in the stiction region. The restoring effects of the elastic elements were determined by measurements.
\end{abstract}

Keywords: pneumatic system, stiffness, friction, stiction

\section{INTRODUCTION}

The increasing positioning accuracy of pneumatic actuating units not only at the final positions, but also along the whole actuating track became an important research field during the last two decades of the 20th century. The investigations to improve the control algorithms of these so called "servo-pneumatic" systems usually in the centre of attention since the robust control of these systems is a rather complicated task because of the physical complexity of pneumatic systems. That is why, during the last couple of years many research groups are occupied with the dynamical analysis of servo pneumatic systems and are suggesting diverse algorithms to improve the positioning accuracy and the stiffness of these systems.

Based on new measurement methods and resulting statements, it is possible to increase the positioning accuracy and the dynamic properties of servo pneumatic systems. Considering this not only the improvement of control quality can be the aim of the recent investigations, but also the search for the reserves within the pneumatic system is a real life research task. According to this statement the dynamical modelling of complex pneumatic systems and the clarification of the relationships among the parameters are in the centre of the presented research.

The stiffness of the system plays an important role in the dynamic properties, because among other things this feature determines the resistance against forces and other disturbances when keeping a position. In contrast to mechanical propulsion systems (gearbox, toothed belt gear, worm gear, etc.) or hydraulic systems [1], pneumatic systems have a special feature because of the low value of the stiffness [2], [3].

The analysis of the pressure and the capacity of the chamber(s) is an important factor in case of rigid cylinder wall [3] and pneumatic muscles [4] as well. However the fundamental equations for stiffness description are well known, and many control strategies are developed based on the control of pneumatic stiffness [5], [6] but, the resulting spring stiffness, or the returning pressure of a double acting cylinder is not discussed. The focus of the research presented is the returning pressure determination of pneumatic cylinders.

\section{MATERIALS AND METHODS}

The two most important factors of passive resistance - without any correction by valve actuation - to external forces are the resilience of the pressure changes and the restoring action of the seals in the stiction 
region for small piston displacements. Several literature [2] [7] references indicate that the spring-like effect of seals in a close distance of the piston position causes forces acting against the load forces until the moment when the adhesion ceases.

The studies were carried out for the analysis of a rodless double-acting pneumatic cylinder type OSP-P210-032-700 as shown on Fig. 1. The resulting restoring force equations from changes in pressure changes in chambers are valid for other double-acting cylinders, too.

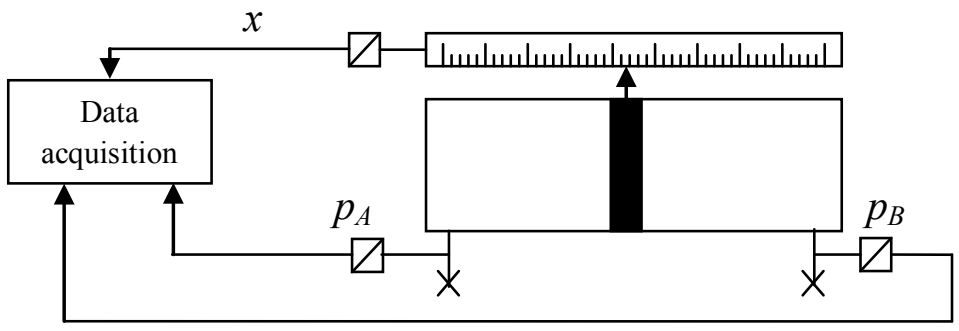

Figure 1. Measurement setup

\section{RESULTS}

Passive resistance of pneumatic cylinders against external forces can be defined by the resulting of two factors: resilience of the pressure changes and the restoring action force of the seals in the stiction region must be taken into consideration. Furthermore these properties are investigated.

\subsection{Pressure changes in the chambers as a function of piston displacement}

The chambers of the pneumatic cylinders behave as springs directed against one another. The stiffness of a material or structure is higher when the deformation from a given load is smaller. The modulus of elasticity or Young's-modulus $E$ describes stiffness and elasticity. The connection for the description of the strain $\varepsilon$ with respect to a stress $\sigma$ for a load on the structure is:

$$
E=\frac{\partial \sigma}{\partial \varepsilon}
$$

In the denominator strain $\varepsilon$ as the relative displacement can be described as:

$$
\varepsilon=\frac{\Delta l}{l_{0}}
$$

For linearly elastic materials (like many metals), Hooke's Law states, that the stress of a body to the strain in the elastic range is load independent. The modulus of elasticity in gases depends not only on the material properties, but also on the load, therefore further analysis is necessary.

The transformation of (1) is valid for pneumatic systems, as well. However, it is known as bulk modulus $B$ instead of Young's-modulus $E$. The nominator of (1) represents the tensile or compressive stress normal to the plane usually denoted "normal stress" and can be expressed as:

$$
\sigma=\frac{F}{A}
$$


Equation (3) describes the acting force $F$ on the piston cross section $A$, which corresponds the pressure $p$ for a pneumatic cylinder. For pneumatic cylinder with a piston surface $A$ of this chamber is equivalent to a specific change in length, so equation (1) is as follows:

$$
B=x \cdot \frac{\partial p}{\partial x}
$$

To derive the bulk modulus a description of the relationship between volume change and the change in pressure is needed. It can be assumed that the cylinder cross-section is constant along the stroke length $l_{k}$, the volume of the chambers varies directly proportional with the displacement $x$. In the polytrophic process equation of ideal gases the volume $V$ of the chamber can be written as a function of chamber cross section area and chamber length:

$$
p \cdot V^{n}=p \cdot A^{n} \cdot x^{n}=\text { const } .
$$

In case of isentropic processes, when there is no heat exchange between the gas and the wall of the cylinder, as it is in the presented case, the polytrophic exponent is $n=\kappa=1,4$. If the piston is moving slowly the heat exchange generated through the walls of the chamber is unavoidable, in which case the polytrophic exponent value $n=1$ for isothermal processes is used. As it is derived in [3], the Bulk modulus can be expressed from the total derivative of (5) as:

$$
B=n \cdot p
$$

Equation (6) shows that the bulk modulus of a double-acting pneumatic system (chamber) is not constant but increases linearly with the pressure. However a question arises, namely how big is the restoring force for a certain cylinder?

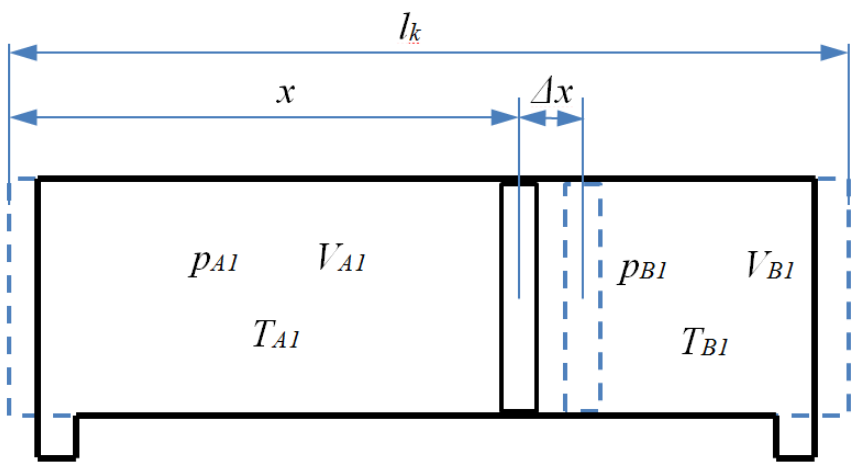

Figure 2. Principle diagram of the Pneumatic Cylinder state variables

Holding a position $x$ means that the piston is in the appropriate position without any outside action, that means, the valves are closed. Accordingly the cylinder chambers can be considered as a closed system. For determination of the resulting spring stiffness along the stroke length of the double-acting cylinder the pressure changes should be specified in both chambers separately for small displacement $\Delta \mathrm{x}$. Cylinders have addition dead volumes in connecting tubes and covers. In the calculation of the state change of compressed air these volumes must be taken into consideration as it is shown by the dashed line on Fig. 2 . 
The restoring force $\Delta F$ against displacement of the piston is proportional. The stiffness of the chambers is derived from the polytrophic state equation for small displacements $\Delta x$ :

$$
\begin{gathered}
p_{A 1} \cdot A_{A}^{n} \cdot x^{n}=p_{A 2} \cdot A_{A}^{n} \cdot(x+\Delta x)^{n} \\
p_{B 1} \cdot A_{B}^{n} \cdot\left(l_{k}-x\right)^{n}=p_{B 2} \cdot A_{B}^{n} \cdot\left(l_{k}-x-\Delta x\right)^{n}
\end{gathered}
$$

The cross section of the pistons for both chambers does not change it can be used as a simplification. It is an important result that the chamber pressure does not depend on the piston cross-sectional area, i.e. the derivation of the change in chamber pressures applies to cylinders with piston rod and for rodless versions. The goal is to express the chamber pressure changes $\Delta p$ as a function of the piston position $x$ :

$$
\begin{gathered}
\Delta p_{A}=p_{A 2}-p_{A 1}=p_{A 1} \cdot \frac{x^{n}-(x+\Delta x)^{n}}{(x+\Delta x)^{n}} \\
\Delta p_{B}=p_{B 2}-p_{B 1}=p_{B 1} \cdot \frac{\left(l_{k}-x\right)^{n}-\left(l_{k}-x+\Delta x\right)^{n}}{\left(l_{k}-x+\Delta x\right)^{n}}
\end{gathered}
$$

The numerator of (6) resp. (7) can be merged using Taylor series of $(x+\Delta x)^{n}$ :

$$
f(x)=(x+\Delta x)^{n}=x^{n}+n \cdot x^{n-1} \cdot \Delta x+\frac{n \cdot(n-1) \cdot x^{n-2}}{2} \cdot \Delta x^{2}+\ldots
$$

In the denominators of (9) and (10) $\Delta x$ is relatively small compared to other coefficients, accordingly the simplifications $(x+\Delta x)^{n}=x^{n}$, resp. $\left(l_{k}-x-\Delta x\right)^{n}=\left(l_{k}-x\right)^{n}$ are possible. The resulting pressure change, or returning pressure $\Delta p_{F}$ of the two chambers:

$$
\Delta p_{F}=\Delta p_{B}-\Delta p_{A}=p_{B 1} \cdot n \cdot \frac{\Delta x}{\left(l_{k}-x\right)}+p_{A 1} \cdot n \cdot \frac{\Delta x}{x}
$$

When there is no external force exerted on the piston, the pressures of the chambers are equal $\left(p_{A l}=p_{B I}\right)$ :

$$
\Delta p_{F}=n \cdot \Delta x \cdot \frac{p_{A 1} \cdot\left(l_{k}-x\right)+p_{A 1} \cdot x}{x \cdot\left(l_{k}-x\right)}=p_{A 1} \cdot n \cdot \Delta x \cdot \frac{l_{k}}{x \cdot\left(l_{k}-x\right)}
$$

The returning pressure $\Delta p_{F}$ can be expressed as a function of the chamber pressure $p_{A 1}$ or $p_{B 1}$ :

$$
\frac{\Delta p_{F}}{p_{A 1}}=n \cdot \Delta x \cdot \frac{l_{k}}{x \cdot\left(l_{k}-x\right)}
$$

Equation (14) gives information of the relative return pressure in case of small displacements. Exact results cannot be obtained, because the state change can range from isothermal to adiabatic in relation with the heat exchange of the gas inside the chamber. The polytrophic exponent varies between 1..1.4 accordingly. However, according to the results the adiabatic state change approximates the process well.

The equation is not suitable for greater displacements. The reason is that for major displacements the exponential function of pressure change is described by a linear equation due the simplification by Taylor series. (11). Relative pressure changes caused by small piston displacements are shown on Fig. 3. 


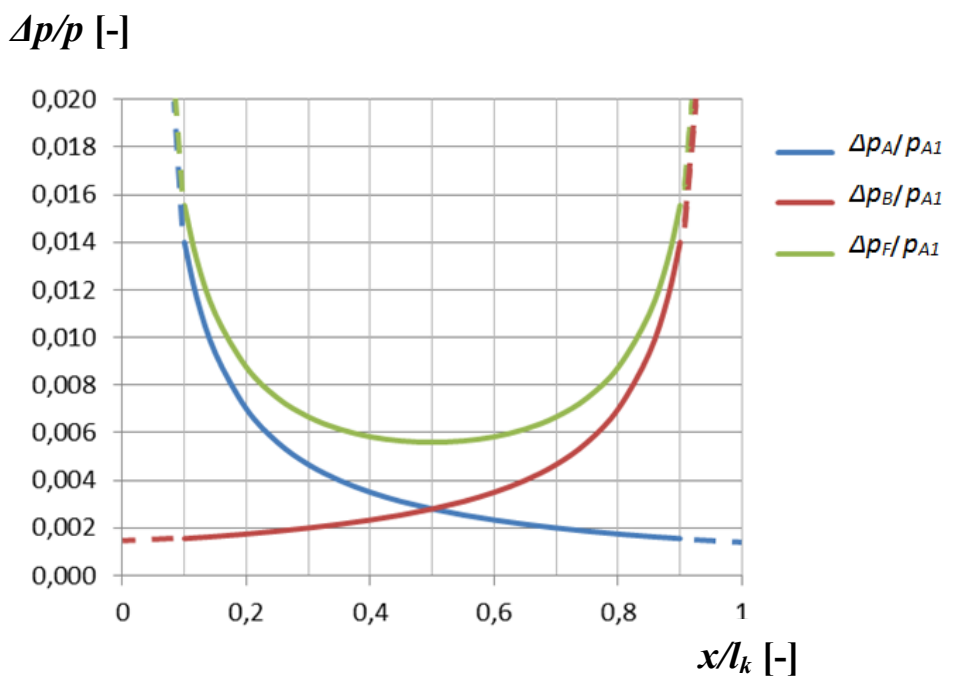

Figure 3. Relative return pressure ( $(\mathrm{p} / \mathrm{p})$ of the chambers at $0.1 \%$ relative displacement in relation to the total stroke

Figure 3 shows the relative return pressures of the chambers at $0.1 \%$ relative displacement in relation to the total stroke, which corresponds to a $1 \mathrm{~mm}$ displacement of a cylinder with a stroke length of $1 \mathrm{~m}$. The dashed line represents the extended stroke length considered as dead volumes. Equation (15) determines the return force of the pressurized air for rodless cylinders.

$$
\Delta F=\Delta p_{F} \cdot A=p_{A 1} \cdot n \cdot \Delta x \cdot \frac{l_{k}}{x \cdot\left(l_{k}-x\right)}
$$

When the task is not just positioning, but a certain force $F$ has to provide by the cylinder the chambers must have different pressures. In this case manifestly $p_{A I} \neq p_{B 1}$, so the simplification applied in (13) is not possible.

\subsection{Restoring action force of the seals in the stiction region}

Friction is a central phenomenon in most technical applications. The precise friction model is important for friction compensation in many control strategies. For a long time friction of various systems - among others for pneumatic systems - was described by Coulomb and viscous friction model added to Stribeck's term in the sliding regime. However these models in case of accurate positioning significant slow movements - especially zero velocity -were not appropriate. One of the first descriptions of this phenomenon, so called pre-sliding region is well known as Dahl's theory [8]. It proposed that the relationship between frictional force and position would be analogous to a stress-strain curve, completed with hysteresis like plastic deformation of materials [9].Since then, several models have been developed which are suitable for the description of slow movements. This article has not the task to compare different friction models, but to determine the limits of stiction regime in case of given pneumatic cylinder, and to discuss the effects of the mentioned factors.

The force-displacement characteristics of the rodless pneumatic cylinder OSP-P-210-032-700 were measured on chamber pressures from atmospheric pressure to overpressure of 6 bars. In addition the investigations of the rodless pneumatic cylinder were extended at horizontal cylinder arrangement on various load weights. The chambers $p_{A}$ and $p_{B}$ had the same pressure value, so the results do not influenced 
by pressure difference of the chambers. The measured results indicate the slope of the curves which depends only a little on the chamber pressure and on the load in the stiction area.

The width of the stiction area is obviously proportional to those, the exerted force can be maximal $15 . .20 \mathrm{~N}$ to stay within the stiction area. One of these trajectories shows Fig 4 . The slope of the curve is about 40 $\mathrm{N} / \mathrm{mm}$. Due to parameter changes and measurement uncertainties a more precise calculation of the slope makes no sense.

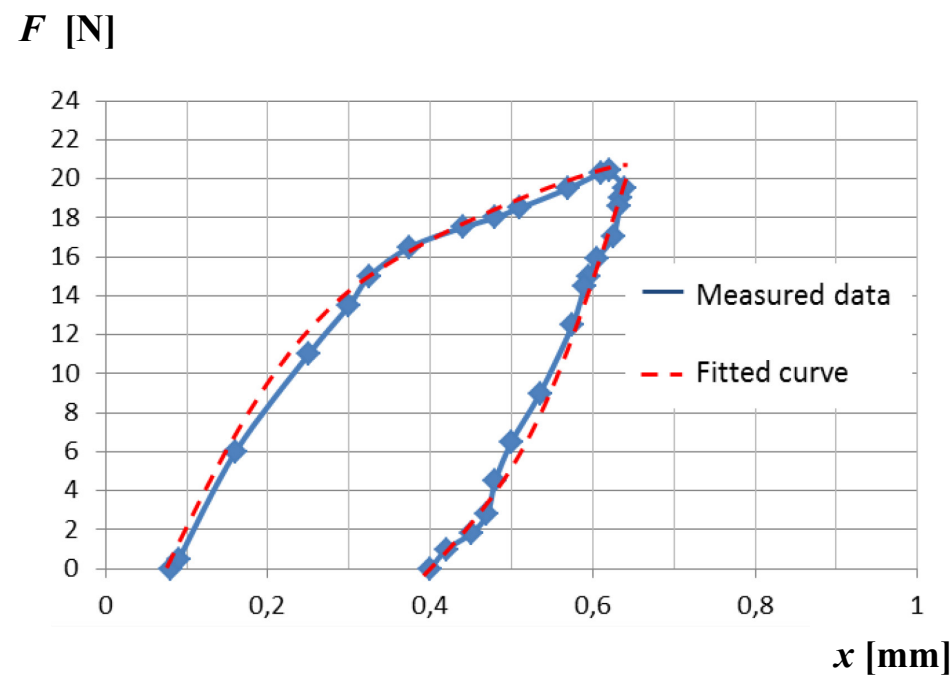

Figure 4. Force-displacement characteristics of cylinder OSP-P-210-032-700 at 6 bars chamber pressure

\section{DISCUSSION}

The investigated restoring action force of the seals is typically $20 \mathrm{~N}$ and causes a displacement of $0.5 \mathrm{~mm}$ magnitude in the stiction region. For greater forces the friction gets in the sliding friction regime, the piston gets to move continuously. The restoring force due to pressure change of the chambers - in case of piston displacement for the mentioned $0,5 \mathrm{~mm}$ - from (15) results a magnitude of $5 \mathrm{~N}$ in the middle of the stroke, which can be risen approximately until $20 \mathrm{~N}$ at the end of it. It can be stated clear that the effect of the restoring action force due to chamber pressure change respect to the restoring force of the seals in the stiction region is marginal.

\section{CONCLUSIONS}

Double-acting pneumatic cylinders have two passive factors of resistance against external forces. For small displacements in the stiction region the restoring action force of the seals is dominant. The resilience of the pressure change prevails only for larger displacements, typically out of the stiction regime. The border of the two effects depends on the stiction limit, which is not clearly definable. It depends on the material, construction parameters and pretension of the seals, lubrication, stay duration of the piston, and the pressure rate of the action. During the measurements there was not any external guide equipped on the pneumatic actuator, which friction properties can influence the whole pneumatic cylinder.

The resilience of the cylinder against the acting forces should be examined in a different way for small and for large displacements. For small displacements (micrometre range) the stiffness of the pneumatic cylinder cannot be increased significantly with increasing of the chamber pressures. It can only be influenced by the design, and optimization of the seals and the guide. 


\section{ACKNOWLEDGEMENT}

This work is connected to the scientific program of the " Development of quality-oriented and harmonized $\mathrm{R}+\mathrm{D}+\mathrm{I}$ strategy and functional model at BME" project. This project is supported by the New Hungary Development Plan (Project ID: TÁMOP-4.2.1/B-09/1/KMR-2010-0002).

\section{REFERENCES}

[1] Szép E., Stiffness of Throttled Hydrostatic Transmissions, Periodica Polytechnica Ser. Mech. Eng., Vol. 10, No. 1., pp. 59-68, 1996.

[2] M. J. Meuser, Nichtlineare Regelung pneumatischer Antriebe, Shaker Verlag, 2010.

[3] H. Murrenhoff, Grundlagen der Fluidtechnik Teil 2 Pneumatik, Shaker Verlag, 2006.

[4] Sárosi J., Bíró I., Németh J. Cveticanin L.: Dynamic Modelling of a Pneumatic Muscle Actuator with Two-direction Motion, Mechanism and Machine Theory, Vol. 85, pp. 25-34, 2015.

[5] K. Széll, A. Czmerk, P. Korondi, „Friction with Hysteresis Loop Modeled by Tensor Product,” Automatika, Vol. 55, No. 4., pp. 463-473, 2014.

[6] Behzad Taheri, David Case, and Edmond Richer, Force and Stiffness Backstepping-Sliding Mode Controller for Pneumatic Cylinders, Mechatronics, IEEE/ASME Transactions on Mechatronics, Vol.19, Issue: 6 pp. 1799-1809., 2014.

[7] B. M. Nouri és M. B. Saudi, Experimental Modelling and Identification of Compressible Flow through Proportional Directional Control Valves, Universal Journal of Control and Automation, Vol. 1., pp. 413., 2014.

[8] P.R. Dahl., A solid friction model. The Aerospace Corporation. Technical report, 1968.

[9] B. Armstrong-Helouvry, P. Dupont és C. de Vit, „A Survey of Models, Analysis Tools and Compensation. Methods for the Control of Machines with Friction," Automatica, Vol. 30, No. 7, pp. 1083-1138, 1994. 